\title{
On the relations of general homogeneous spaces and the two-wavelet localization operators
}

\section{Olya Dokht Sajadi Rad ${ }^{1} \cdot$ Rajab Ali Kamyabi Gol ${ }^{2} \cdot$ Fatemeh Esmaeelzadeh $^{3}$}

Received: 12 August 2020 / Revised: 14 November 2020 / Accepted: 23 November 2020 /

Published online: 9 March 2021

(c) The Author(s) 2021

\begin{abstract}
In this note, the two-wavelet localization operator for square integrable representation of a general homogeneous space is defined. Then among other things, the boundedness properties of this operator is investigated. In particular, it is shown that it is in the Schatten p-class.
\end{abstract}

Keywords Strongly quasi invariant measure - Two-wavelet Localization operator · Homogeneous space $\cdot$ Continuous wavelet transform

\section{Introduction and preliminaries}

The name of localization operator goes back to 1988, when I. Daubechies first used these operators as a mathematical tool to localize a signal on the time frequency plane. Recently, localization operators have been a subject of study in quantum mechanics; in PDE and signal analysis (see [1,3,10]). Wong [12] has studied the localization operators in the setting of homogeneous spaces with a $G$-invariant measure (see also [2]). The localization operators in the setting of homogeneous space with an admissible wavelet on general homogeneous space were studied in [11]. Also the two-wavelet localization operators on the homogeneous space $\mathrm{G} / \mathrm{H}$ has been investigated in $[4,7$, 8], where $\mathrm{G} / \mathrm{H}$ was equipped with a relatively invariant measure. In this paper, we

$凶 \quad$ Rajab Ali Kamyabi Gol

kamyabi@um.ac.ir

Olya Dokht Sajadi Rad

sajadyrad@stu.um.ac.ir

Fatemeh Esmaeelzadeh

esmaeelzadeh@bojnourdiau.ac.ir

1 Ferdowsi University of Mashhad, P. O. Box 1159, Mashhad 91775, Iran

2 Department of Pure Mathematics, Center of Excellence in Analysis on Algebraic Structures

(CEAAS), Ferdowsi University of Mashhad, P. O. Box 1159, Mashhad 91775, Iran

3 Department of Mathematics, Bojnourd Branch, Islamic Azad University, Bojnourd, Iran

Birkhäuser 
continue our investigation on the localization operators with two admissible wavelets in a completely different and more general approach, by considering a strongly quasi invariant measure on the homogeneous space $\mathrm{G} / \mathrm{H}$. The purpose of this paper is to explore and develop certain results concerning two wavelet localization operators in the setting of general homogeneous spaces. At this point for reader convenince and fixing our notations, we recall some remarkable pointers of Radon measures on homogeneous spaces.

Let $\mathrm{G}$ be a locally compact group and $\mathrm{H}$ be a closed subgroup of $\mathrm{G}$. Consider $G / H$ as a homogeneous space equipped with a strongly quasi invariant measure $v$. Note that $\mathrm{G}$ acts from the left on $G / H$ in a natural way. We recall that a non-zero positive Radon measure $v$ is strongly quasi invariant if there is a continuous function $\kappa: G \times G / H \rightarrow(0, \infty)$ such that

$$
d v_{g}(k H)=\kappa(g, k H) d \nu(k H),
$$

for all $g, k \in G$. If the function $\kappa(g, \cdot)$ reduces to constant, then $v$ is called relatively invariant under $G$. It is well known that any homogeneous space $G / H$ possesses a strongly quasi invariant measure and all such measures are constructed by a rhofunction $\rho$. We recall that a rho-function for the pair $(G, H)$ is defined to be a continuous function $\rho: G \rightarrow(0, \infty)$ which satisfies

$$
\rho(g h)=\frac{\Delta_{H}(h)}{\Delta_{G}(h)} \rho(g) \quad(g \in G, h \in H),
$$

where $\Delta_{G}, \Delta_{H}$ are the modular functions on $G$ and $H$, respectively (see [6]). For any pair $(\mathrm{G}, \mathrm{H})$ and each rho-function $\rho$, there is a strongly quasi invariant measure $v$ on $G / H$ such that

$$
d \nu_{g}(k H)=\kappa(g, k H) d \nu(k H) \quad(g, k \in G),
$$

in which

$$
\kappa(g, k H)=\frac{\rho(g k)}{\rho(k)} .
$$

It is easy to see that

$$
\begin{aligned}
\kappa\left(g_{1} g_{2}, x\right) & =\kappa\left(g_{1}, g_{2} x\right) \kappa\left(g_{2}, x\right), \\
\kappa(e, x) & =1 .
\end{aligned}
$$

A positive Radon measure $v$ on $G / H$ is said to be G-invariant if $v_{g}=v$ for all $g \in \mathrm{G}$. It is well known that $G / H$ admits a $G$-invariant measure when $H$ is compact. (for a detailed account of Homogeneous spaces, the reader is referred to $[6,7,9])$.

Now, we recall the basic concepts of continuous unitary representations and continuous wavelet transform on homogeneous spaces [5]. For a Hilbert space $\mathcal{H}$, let 
$U(\mathcal{H})$ denote the group of unitary operators on $\mathcal{H}$ equipped with the strong operator topology.

A continuous map

$$
\begin{aligned}
\varrho: G / H & \longrightarrow U(\mathcal{H}) \\
g H & \longrightarrow\langle\varrho(g H) x, y\rangle
\end{aligned}
$$

that $\varrho(g k H)=\varrho(g H) \varrho(k H), \varrho\left(g^{-1} H\right)=\varrho(g H)^{*}$ for each $x, y \in \mathcal{H}$ and $g, k \in G$, is called a continuous unitary representation of homogeneous space $\mathrm{G} / \mathrm{H}$. Moreover a closed subspace $\mathrm{M}$ of $\mathcal{H}$ is said to be invariant with respect to $\varrho$ if $\varrho(g H) M \subseteq M$, for all $g \in G$. A continuous unitary representation $\varrho$ is said to be irreducible if the only invariant subspaces of $\mathcal{H}$ are $\{0\}$ and $\mathcal{H}$.

Note that, there is a significant relationship between the representations of homogeneous space $\mathrm{G} / \mathrm{H}$ and representations of group $\mathrm{G}$. That is, if $\varrho$ is a representation of $\mathrm{G} / \mathrm{H}$, then it defines a representation $\sigma$ of $\mathrm{G}$ in which the subgroup $\mathrm{H}$ is considered to be contained in the kernel of $\sigma$. Conversely, any representation $\sigma$ of $\mathrm{G}$ which is trivial on $\mathrm{H}$ induces a representation $\varrho$ of $\mathrm{G} / \mathrm{H}$ by letting $\varrho(g H)=\sigma(g)$.

Let $\varrho$ be a unitary irreducible representation of $\mathrm{G} / \mathrm{H}$ on a Hilbert space $\mathcal{H}$. A vector $\eta \in \mathcal{H}$ is said to be admissible if

$$
\int_{G / H} \kappa\left(g^{-1}, g H\right)|\langle\eta, \varrho(g H) \eta\rangle|^{2} d \nu(g H)<\infty .
$$

An admissible vector $\eta \in \mathcal{H}$ is called admissible wavelet if $\|\eta\|=1$.

Note that (1.3) is well-defined [11].

For a representation $\varrho$ of $\mathrm{G} / \mathrm{H}$ on Hilbert space $\mathcal{H}$ and an admissible wavelet $\eta$ for $\varrho$, the continuous wavelet transform associated with the admissible wavelet $\eta$ is defined as the linear operator $\Omega_{\eta}$ from $\mathcal{H}$ into the space of continuous functions of $G / H$,

$$
\Omega_{\eta} \Psi(g H)=\left(\frac{\kappa\left(g^{-1}, g H\right)}{\chi_{\eta}}\right)^{\frac{1}{2}}\langle\Psi, \varrho(g H) \eta\rangle,
$$

for all $\Psi \in \mathcal{H}, g \in G$ where

$$
\chi_{\eta}:=\int_{G / H} \kappa\left(g^{-1}, g H\right)|\langle\eta, \varrho(g H) \eta\rangle|^{2} d \nu(g H),
$$

is called the wavelet constant associated to $\eta$ in $\mathcal{H}$.

In the following theorem, we collect some properties of a square integrable representation of homogeneous $G / H$ on a Hilbert space $\mathcal{H}$ with two admissible wavelets that are needed in the sequel.

Theorem 1.1 Let $\varrho$ be a square integrable representation of $G / H$ on the Hilbert space $\mathcal{H}$ and $\eta, \xi$ be two admissible wavelets for $\varrho$. Then the following holds. 
(i) For all $x, y$ in $\mathcal{H}$

$$
\int_{G / H} \frac{\kappa\left(g^{-1}, g H\right)}{\sqrt{\chi_{\eta} \chi \xi}}\langle x, \varrho(g H) \eta\rangle\langle\varrho(g H) \xi, y\rangle d \nu(g H)=\chi_{\eta, \xi}\langle x, y\rangle,
$$

in which

$$
\chi_{\eta, \xi}=\int_{G / H} \frac{\kappa\left(g^{-1}, g H\right)}{\sqrt{\chi_{\eta} \chi_{\xi}}}\langle\eta, \varrho(g H) \eta\rangle\langle\varrho(g H) \xi, \eta\rangle d \nu(g H),
$$

(ii) There exists a unique self adjoint positive operator $C$ on $\mathcal{H}$ such that:

(a) The set of all admissible wavelets coincide with the domain of $C$.

(b) For all $x, y$ in $\mathcal{H}$,

$$
\int_{G / H} \frac{\kappa\left(g^{-1}, g H\right)}{\sqrt{\chi_{\eta} \chi \xi}}\langle x, \varrho(g H) \eta\rangle\langle\varrho(g H) \xi, y\rangle d \nu(g H)=\langle C \eta, C \xi\rangle\langle x, y\rangle
$$

Proof The proof is the same as Theorem 2.1 of [11].

The $\chi_{\eta, \xi}$ in part(i) is called the two-wavelet constant.

In the rest of the paper, we aim to study the two-wavelet localization operators on a homogeneous space $\mathrm{G} / \mathrm{H}$ equipped with a strongly quasi invariant measure which are related to a continuous wavelet transform. In Sect. 2, a two-wavelet localization operator which is related to continuous wavelet transform (C.W.T) is defined. In this setting, the boundedness properties of two-wavelet localization operators is investigated. In Sect. 3, we show that the two-wavelet localization operators are in p-Schatten class.

\section{Two-wavelet localization operator}

Throughout this section $\mathrm{G}$ is a locally compact group and $\mathrm{H}$ is a compact subgroup of $\mathrm{G}$. Consider $\mathrm{G} / \mathrm{H}$ as a homogeneous space equipped with a strongly quasi invariant measure $v$ which arises from a rho- function $\rho$. Let $\mathcal{H}$ be a separable Hilbert space, $\varrho$ a square integrable representation of $\mathrm{G} / \mathrm{H}$ on $\mathcal{H}$ and $\eta, \xi$ are admissible wavelets for $\varrho$. In this section, first, we define a two-localization operator $\Upsilon_{\Theta, \eta, \xi}$ which is related to the C.W.T $\Omega_{\eta}$ for each $\Theta \in L^{p}(G / H), 1 \leq p \leq \infty$ and then we investigate the boundedness properties of this operator. To do so, we need the following definition.

Definition 2.1 Let $\mathcal{H}$ be a Hilbert space and $\varrho$ a square integrable representation of $\mathrm{G} / \mathrm{H}$ on $\mathcal{H}$ with two admissible wavelets $\eta, \xi$. A two-wavelet localization operator is a linear operator $\Upsilon_{\Theta, \eta, \xi}$ on $\mathcal{H}$ as

$$
\begin{aligned}
\left\langle\Upsilon_{\Theta, \eta, \xi} x, y\right\rangle= & \int_{G / H} \frac{\kappa\left(g^{-1}, g H\right)}{\chi_{\eta, \xi}} \Theta(g H) \\
& \langle x, \varrho(g H) \eta\rangle\langle\varrho(g H) \xi, y\rangle d \nu(g H),
\end{aligned}
$$


for all $\Theta \in L^{p}(G / H)$ and $x, y \in \mathcal{H}$, where $\chi_{\eta, \xi}$ is the two-wavelet constant.

Now we show that the two-wavelet localization operator is bounded for $\Theta \in$ $L^{p}(G / H), 1 \leq p \leq \infty$.

The following proposition shows that $\Upsilon_{\Theta, \eta, \xi}$ is bounded for $\Theta \in L^{\infty}(G / H)$.

Proposition 2.2 By considering the notations above, let $\Theta \in L^{\infty}(G / H)$; Then $\Upsilon_{\Theta, \eta, \xi}$ is a bounded linear operator and

$$
\left\|\Upsilon_{\Theta, \eta, \xi}\right\| \leq \frac{\left(\chi_{\eta} \chi_{\xi}\right)^{\frac{1}{2}}}{\left|\chi_{\eta, \xi}\right|}\|\Theta\|_{\infty},
$$

in which $\eta$ and $\xi$ are two admissible wavelets.

Proof To do this, using the reconstruction formula and the Schwartz inequality, we get,

$$
\begin{aligned}
\left|\left\langle\Upsilon_{\Theta, \eta, \xi} x, y\right\rangle\right| & \leq \frac{1}{\left|\chi_{\eta, \xi}\right|} \int_{G / H} \kappa\left(g^{-1}, g H\right)|\Theta(g H) \|\langle x, \varrho(g H) \eta\rangle||\langle\varrho(g H) \xi, y\rangle| d \nu(g H) \\
& \left.\leq \frac{1}{\left|\chi_{\eta, \xi}\right|}\|\Theta\|_{\infty} \int_{G / H} \kappa\left(g^{-1}, g H\right)|\langle x, \varrho(g H) \eta\rangle|^{2} d \nu(g H)\right)^{\frac{1}{2}} \\
& \left(\int_{G / H} \kappa\left(g^{-1}, g H\right)|\langle\varrho(g H) \xi, y\rangle|^{2} d \nu(g H)\right)^{\frac{1}{2}} \\
& \leq \frac{\left(\chi_{\eta} \chi_{\xi}\right)^{\frac{1}{2}}}{\left|\chi_{\eta, \xi}\right|}\|\Theta\|_{\infty}\left(\int_{G / H}\left|\Omega_{\eta}(x)\right|^{2} d \nu\right)^{\frac{1}{2}}\left(\int_{G / H}\left|\overline{\Omega_{\eta}(y)}\right|^{2} d \nu(g H)\right)^{\frac{1}{2}} \\
& \leq \frac{\left(\chi_{\eta} \chi_{\xi}\right)^{\frac{1}{2}}}{\left|\chi_{\eta, \xi}\right|}\|\Theta\|_{\infty}\|x\|\|y\|,
\end{aligned} .
$$

So for $\Theta \in L^{\infty}(G / H)$,

$$
\left\|\Upsilon_{\Theta, \eta, \xi}\right\| \leq \frac{\left(\chi_{\eta} \chi_{\xi}\right)^{\frac{1}{2}}}{\left|\chi_{\eta, \xi}\right|}\|\Theta\|_{\infty} .
$$

As mentioned earlier, $\mathrm{G} / \mathrm{H}$ admits such a $\mathrm{G}$-invariant measure when $\mathrm{H}$ is compact. Now we show that $\Upsilon_{\Theta, \eta, \xi}$ is bounded for $\Theta \in L^{1}(G / H)$ in which $\mathrm{G} / \mathrm{H}$ is equipped with a G-invariant measure $\nu^{\prime}$.

Proposition 2.3 With the above notations, let $\Theta \in L^{p}(G / H), 1 \leq p<\infty$. Then $\Upsilon_{\Theta, \eta, \xi}$ is a bounded linear operator and

$$
\left\|\Upsilon_{\Theta, \eta, \xi}\right\| \leq \frac{\rho(e)^{\frac{1}{p}}}{\left|\chi_{\eta, \xi}\right|}\left(\chi_{\eta} \chi_{\xi}\right)^{\frac{1}{2}\left(1-\frac{1}{p}\right)\|\Theta\|_{p}}
$$


Proof The proof is shown in two steps.

In the first step we show that if $\Theta \in L^{1}(G / H)$ then $\left\|\Upsilon_{\Theta, \eta, \xi}\right\| \leq \frac{\rho(e)}{\left|\chi_{\eta, \xi}\right|}\|\Theta\|_{1}$.

In the second step we show that if $\Theta \in L^{p}(G / H), 1<p<\infty$. then

$$
\left\|\Upsilon_{\Theta, \eta, \xi}\right\| \leq \frac{\rho(e)^{\frac{1}{p}}}{\left|\chi_{\eta, \xi}\right|}\left(\chi_{\eta} \chi_{\xi}\right)^{\frac{1}{2}\left(1-\frac{1}{p}\right)\|\Theta\|_{p}}
$$

Let $\Theta \in L^{1}(G / H)$. Consider G/H with a G-invariant measure $v^{\prime}$ which arises from rho-function $\rho^{\prime} \equiv 1$ [6]. Note that since $\mathrm{H}$ is compact, $\mathrm{G} / \mathrm{H}$ admits such a $\mathrm{G}$-invariant measure $v^{\prime}$ which is equivalent to $v$, that is $\frac{d v}{d v^{\prime}}=\varphi$ in which $\varphi(g H)=\frac{\rho(g)}{\rho^{\prime}(g)}$, where $v$ is the strongly quasi invariant measure which arises from rho-function $\rho$. Thus

$$
\begin{aligned}
& \left|\left\langle\Upsilon_{\Theta, \eta, \xi} x, y\right\rangle\right| \\
& \quad \leq \frac{1}{\mid \chi_{\eta, \xi \mid}} \int_{G / H} \kappa\left(g^{-1}, g H\right)|\Theta(g H) \|\langle x, \varrho(g H) \eta\rangle||\langle\varrho(g H) \xi, y\rangle| d \nu(g H) \\
& \leq \frac{1}{\left|\chi_{\eta}, \xi\right|} \int_{G / H} \frac{\rho(e)}{\varphi(g H)}|\Theta(g H)\|\langle x, \varrho(g H) \eta\rangle\|\langle\varrho(g H) \xi, y\rangle| \varphi(g H) d \nu^{\prime}(g H) \\
& \quad \leq \frac{1}{\left|\chi_{\eta, \xi}\right|} \int_{G / H} \rho(e)\|\| x\|\| y \||\Theta(g H)| d \nu^{\prime}(g H) \\
& \leq \frac{\rho(e)}{\left|\chi_{\eta, \xi}\right|}\|\Theta\|_{1}\|\| x\|\| y \|,
\end{aligned}
$$

where $\|\Theta\|_{1}=\int_{G / H}|\Theta(g H)| d \nu^{\prime}(g H)$.

Now through the use of the Reisz Thorin Theorem, we shows that if $\Theta \in$ $L^{p}(G / H), 1<p<\infty$, then there exists a unique bounded linear operator $\Upsilon_{\Theta, \eta, \xi}: \mathcal{H} \longrightarrow \mathcal{H}$ such that

$$
\left\|\Upsilon_{\Theta, \eta, \xi}\right\| \leq \frac{\rho(e)^{\frac{1}{p}}}{\left|\chi_{\eta, \xi}\right|}\left(\chi_{\eta} \chi_{\xi}\right)^{\frac{1}{2}\left(1-\frac{1}{p}\right)\|\Theta\|_{p}}
$$

where $\Upsilon_{\Theta, \eta, \xi}$ is given in (2.1) for a simple function $\Theta$ on $\mathrm{G} / \mathrm{H}$ for which

$$
v(\{g H \in G / H ; \Theta(g H) \neq 0\})<\infty .
$$

Let $\Gamma: \mathcal{H} \longrightarrow L^{2}\left(R^{n}\right)$ be a unitary operator and $\Theta \in L^{1}(G / H)$. Then the linear operator $\Upsilon_{\Theta, \eta, \xi}: L^{2}\left(R^{n}\right) \longrightarrow L^{2}\left(R^{n}\right)$ defined by

$$
\Upsilon^{\sim}{ }_{\Theta, \eta, \xi}=\Gamma \Upsilon_{\Theta, \eta, \xi} \Gamma^{-1}
$$

is bounded and $\left\|\Upsilon^{\sim} \sim_{\Theta, \eta, \xi}\right\| \leq \frac{\rho(e)}{\left|\chi_{\eta, \xi}\right|}\|\Theta\|_{1}$. If $\Theta \in L^{\infty}(G / H)$, then the linear operator $\Upsilon \sim \sim_{\Theta, \eta, \xi}$ on $L^{2}\left(R^{n}\right)$ is bounded and $\left\|\Upsilon \sim_{\Theta, \eta, \xi}\right\| \leq \frac{\left(\chi_{\eta} \chi_{\xi}\right)^{\frac{1}{2}}}{\left|\chi_{\eta}, \xi\right|}\|\Theta\|_{\infty}$. 
Denote by $S$ the set of all simple functions $\Theta$ on $\mathrm{G} / \mathrm{H}$ which satisfy the condition (2.4). Let $g \in L^{2}\left(R^{n}\right)$ and $\Phi_{g}$ be a linear transformation from $S$ into the set of all Lebesgue measurable function on $R^{n}$ defined as $\Phi_{g}(\Theta)=\Upsilon^{\sim}{ }_{\Theta, \eta, \xi}(g)$. Then for all $\Theta \in L^{1}(G / H)$

$$
\left\|\Phi_{g}(\Theta)\right\|_{2}=\left\|\Upsilon^{\sim}{ }_{\Theta, \eta, \xi}(g)\right\|_{2} \leq\left\|\Upsilon^{\sim}{ }_{\Theta, \eta, \xi}\right\|\|g\|_{2} \leq \frac{\rho(e)}{\left|\chi_{\eta, \xi}\right|}\|\Theta\|_{1}\|g\|_{2} .
$$

Similarly for $\Theta \in L^{\infty}(G / H)$,

$$
\left\|\Phi_{g}(\Theta)\right\|_{2} \leq \frac{\left(\chi_{\eta} \chi_{\xi}\right)^{\frac{1}{2}}}{\left|\chi_{\eta, \xi}\right|}\|\Theta\|_{\infty}\|g\|_{2}
$$

Through the Riesz Thorin Interpolation Theorem we get,

$$
\left\|\Upsilon^{\sim}{ }_{\Theta, \eta, \xi}(g)\right\|_{2}=\left\|\Phi_{g}(\Theta)\right\|_{2} \leq \frac{\rho(e)^{\frac{1}{p}}}{\left|\chi_{\eta, \xi}\right|}\left(\chi_{\eta} \chi_{\xi}\right)^{\frac{1}{2}\left(1-\frac{1}{p}\right)}\|\Theta\|_{p}\|g\|_{2} .
$$

So,

$$
\left\|\Upsilon_{\Theta, \eta, \xi}\right\| \leq \frac{\rho(e)^{\frac{1}{p}}}{\left|\chi_{\eta, \xi}\right|}\left(\chi_{\eta} \chi_{\xi}\right)^{\frac{1}{2}\left(1-\frac{1}{p}\right)}\|\Theta\|_{p}
$$

for each $\Theta \in S$.

Now, let $\Theta \in L^{p}(G / H)$, for $1<p<\infty$. Then there exists a sequence $\left\{\Theta_{k}\right\}_{k=1}^{\infty}$ of functions in $S$ such that $\Theta_{k}$ is convergent to $\Theta$ in $L^{p}(G / H)$ as $k \longrightarrow \infty$. Also, $\left\{\Upsilon^{\sim} \Theta_{k}, \eta, \xi\right\}$ is a Cauchy sequence in $B\left(L^{2}\left(\mathbb{R}^{n}\right)\right)$. Indeed,

$$
\left\|\Upsilon^{\sim}{ }_{\Theta_{k}, \eta, \xi}-\Upsilon^{\sim}{ }_{\Theta_{m}, \eta, \xi}\right\| \leq \frac{\rho(e)^{\frac{1}{p}}}{\left|\chi_{\eta, \xi}\right|}\left(\chi_{\eta} \chi_{\xi}\right)^{\frac{1}{2}\left(1-\frac{1}{p}\right)}\left\|\Theta_{k}-\Theta_{m}\right\|_{p} \longrightarrow 0 .
$$

By the completeness of $B\left(L^{2}\left(R^{n}\right)\right)$, there exists a bounded linear operator $\Upsilon \sim_{\Theta, \eta, \xi}$ on $L^{2}\left(R^{n}\right)$ such that $\Upsilon \sim_{\Theta}, \eta, \xi$ converges to $\Upsilon \sim_{\Theta, \eta, \xi}$ in $B\left(L^{2}\left(R^{n}\right)\right)$, in which

$$
\left\|\Upsilon^{\sim}{ }_{\Theta, \eta, \xi}\right\| \leq \frac{\rho(e)^{\frac{1}{p}}}{\left|\chi_{\eta, \xi}\right|}\left(\chi_{\eta} \chi_{\xi}\right)^{\frac{1}{2}\left(1-\frac{1}{p}\right)}\|\Theta\|_{p}
$$

Thus the linear operator $\Upsilon_{\Theta, \eta, \xi}$ is bounded, where $\Upsilon_{\Theta, \eta, \xi}=\Gamma^{-1} \Upsilon_{\Theta, \eta, \xi} \Gamma$ and

$$
\left\|\Upsilon_{\Theta, \eta, \xi}\right\| \leq \frac{\rho(e)^{\frac{1}{p}}}{\left|\chi_{\eta, \xi}\right|}\left(\chi_{\eta} \chi_{\xi \xi}\right)^{\frac{1}{2}\left(1-\frac{1}{p}\right)}\|\Theta\|_{p} .
$$

For the proof of uniqueness, let $\Theta \in L^{p}(G / H), 1<p<\infty$, and suppose that $P_{\Theta, \eta, \xi}$ is another bounded linear operator satisfying (2.3). Let $\Xi: L^{p}(G / H) \longrightarrow B(\mathcal{H})$ be 
the linear operator defined by

$$
\Xi(\Theta)=\Upsilon_{\Theta, \eta, \xi}-P_{\Theta, \eta, \xi}, \quad \Theta \in L^{p}(G / H) .
$$

Then

$$
\|\Xi(\Theta)\| \leq 2 \frac{\rho(e)^{\frac{1}{p}}}{\left|\chi_{\eta, \xi}\right|}\left(\chi_{\eta} \chi_{\xi}\right)^{\frac{1}{2}\left(1-\frac{1}{p}\right)}\|\Theta\|_{p} .
$$

Moreover $\Xi(\Theta)$ is equal to the zero operator on $\mathcal{H}$ for all $\Theta \in S$. Thus, $\Xi$ : $L^{p}(G / H) \longrightarrow B(\mathcal{H})$ is a bounded linear operator that is equal to zero on the dense subspace $S$ of $L^{p}(G / H)$. Therefore $\Upsilon_{\Theta, \eta, \xi}=P_{\Theta, \eta, \xi}$ for all $\Theta \in L^{p}(G / H)$.

\section{Compactness and Schatten p-class}

Let $\mathcal{H}$ be a separable Hilbert space and $B_{1}$ the closed unit ball of $\mathcal{H}$. We recall that a linear operator $T$ on $\mathcal{H}$ is said to be compact if $T\left(B_{1}\right)$ is compact in the norm topology of $\mathcal{H}$. It is well known that a linear operator $T$ on $\mathcal{H}$ is compact if and only if $T\left(B_{1}\right)$ has compact closure in $\mathcal{H}$. Now Suppose $\left\{\alpha_{n}\right\}_{n \in \mathbb{N}}$ and $\left\{\beta_{n}\right\}_{n \in \mathbb{N}}$ are orthonormal sets in $\mathcal{H}$ and $\left\{\lambda_{n}\right\}$ is a sequence of complex numbers tending to 0 . Let $T$ be the linear operator on $\mathcal{H}$ defined by

$$
T \eta=\Sigma_{n=1}^{\infty} \lambda_{n}\left\langle\eta, \alpha_{n}\right\rangle \beta_{n}, \quad \eta \in \mathcal{H}
$$

then $T$ is compact. Note that $\left\{\lambda_{n}\right\}$ are eigenvalues of $|T|=\left(T^{*} T\right)^{\frac{1}{2}}$.

Given $0<p<\infty$, the Schatten p-class of $\mathcal{H}$, denoted by $S_{p}$, is the space of all compact operators $T$ on $\mathcal{H}$ with its singular value sequence $\left\{\lambda_{n}\right\}$ belonging to $l^{p}$, the p-summable sequence space.

In the case, $1 \leq p<\infty, S_{p}$ is a Banach space with the norm

$$
\|T\|_{p}=\left[\Sigma_{n}\left|\lambda_{n}\right|^{p}\right]^{\frac{1}{p}} .
$$

Two special cases are worth mentioning: $S_{1}$ is usually called the trace class and $S_{2}$ is usually called the Hilbert-Schmidt class.

For any $T \in S_{1}$ and any orthonormal basis $\left\{\alpha_{k}\right\}_{k=1}^{\infty}$ of $\mathcal{H}$, we write

$$
\operatorname{tr}(T)=\Sigma_{k=1}^{\infty}\left\langle T \alpha_{k}, \alpha_{k}\right\rangle
$$

and call this value, the trace of $T$. (for more details on Schatten p-classes see [12,13]).

Now we show that the localization operator $\Upsilon_{\Theta, \eta, \xi}$ is in the p-Schatten class. 
Theorem 3.1 Based on the above notations, let $\Theta \in L^{p}(G / H), 1 \leq p \leq \infty$. Then $\Upsilon_{\Theta, \eta, \xi}: \mathcal{H} \longrightarrow \mathcal{H}$ is in $S_{p}$ and

$$
\left\|\Upsilon_{\Theta, \eta, \xi}\right\|_{S_{p}} \leq \frac{\rho(e)^{\frac{1}{p}}}{\left|\chi_{\eta, \xi}\right|}\left(\chi_{\eta} \chi_{\xi}\right)^{\frac{1}{2}\left(1-\frac{1}{p}\right)}\|\Theta\|_{p}
$$

where $\|\Theta\|_{p}^{p}=\int_{G / H}|\Theta(g H)|^{p} d \nu^{\prime}(g H)$ and $\nu^{\prime}$ is a G-invariant measure on $G / H$.

Proof Let $\Theta \in L^{1}(G / H),\left\{\eta_{k}\right\}_{k=1}^{\infty}$ and $\left\{\xi_{k}\right\}_{k=1}^{\infty}$ be any orthonormal set of $\mathcal{H}$ and $\nu^{\prime}$ be a G-invariant measure on $\mathrm{G} / \mathrm{H}$ which arises from $\rho^{\prime}(g) \equiv 1$, we get

$$
\begin{aligned}
\Sigma_{k=1}^{\infty}\left|\left\langle\Upsilon_{\Theta, \eta, \xi} \eta_{k}, \eta_{k}\right\rangle\right| \\
\quad \leq \frac{1}{\left|\chi_{\eta, \xi}\right|} \Sigma_{k=1}^{\infty} \int_{G / H} \kappa\left(g^{-1}, g H\right)|\Theta(g H)|\left|\left\langle\eta_{k}, \varrho(g H) \eta\right\rangle\right|\left|\left\langle\varrho(g H) \xi, \eta_{k}\right\rangle\right| d \nu(g H) \\
\quad \leq \frac{\rho(e)}{\left|\chi_{\eta, \xi}\right|} \int_{G / H}|\Theta(g H)|\left(\Sigma_{k=1}^{\infty}\left|\left\langle\eta_{k}, \varrho(g H) \eta\right\rangle\right|^{2}\right)^{\frac{1}{2}}\left(\Sigma_{k=1}^{\infty}\left|\left\langle\varrho(g H) \xi, \eta_{k}\right\rangle\right|^{2}\right)^{\frac{1}{2}} d \nu^{\prime}(g H) \\
\quad \leq \frac{\rho(e)}{\left|\chi_{\eta, \xi}\right|} \int_{G / H}|\Theta(g H)| d \nu^{\prime}(g H) \\
\quad \leq \frac{\rho(e)}{\left|\chi_{\eta, \xi}\right|}\|\Theta\|_{1}<\infty .
\end{aligned}
$$

So $\Upsilon_{\Theta, \eta, \xi} \in S_{1}$. Now by Proposition 2.2, the two-wavelet localization operator $\Upsilon_{\Theta, \eta, \xi}$ is a bounded linear operator for $\Theta \in L^{\infty}(G / H)$ and

$$
\left\|\Upsilon_{\Theta, \eta, \xi}\right\|_{S_{\infty}} \leq \frac{\left(\chi_{\eta} \chi_{\xi}\right)^{\frac{1}{2}}}{\left|\chi_{\eta, \xi}\right|}\|\Theta\|_{\infty}
$$

Then Reisz Torin Interpolation Theorem [13] implies that the localization operator $\Upsilon_{\Theta, \eta, \xi}$ for $1 \leq p \leq \infty$ is in $S_{p}$.

Here, we obtain a formula for the trace class of the localization operator $\Upsilon_{\Theta, \eta, \xi}$ where $\Theta \in L^{1}(G / H)$.

Theorem 3.2 Let $\Theta \in L^{1}(G / H)$ be a positive function. Then $\operatorname{tr}\left(\Upsilon_{\Theta, \eta, \xi}\right)=$ $\frac{\rho(e)}{\chi_{\eta, \xi}}\langle\xi, \eta\rangle\|\Theta\|_{1}$ in which $v^{\prime}$ is a G-invariant measure on $G / H$.

Proof First note that, since $\mathrm{H}$ is compact, $G / H$ admits a G-invariant measure $v^{\prime}$. Also we equipped $G / H$ with a strongly quasi invariant measure $v$ which arises from a rhofunction $\rho$. These two measures are related as follows, $\frac{d v}{d \nu^{\prime}}=\varphi$, where $\varphi(g H)=\rho(g)$. Now, let $\left\{\eta_{k} ; k=1,2, \ldots\right\}$ be an orthonormal basis for $\mathcal{H}$. Then by using Fubini's 
theorem, Parseval's identity, for $\Theta \in L^{1}(G / H)$ we get

$$
\begin{aligned}
\operatorname{tr}\left(\Upsilon_{\Theta, \eta, \xi}\right)= & \Sigma_{k=1}^{\infty}\left(\Upsilon_{\Theta, \eta, \xi} \eta_{k}, \eta_{k}\right) \\
= & \frac{1}{\chi_{\eta, \xi}} \Sigma_{k=1}^{\infty} \int_{G / H} \kappa\left(g^{-1}, g H\right) \Theta(g H)\left\langle\eta_{k}, \varrho(g H) \eta\right\rangle \\
& \left\langle\varrho(g H) \xi, \eta_{k}\right\rangle \rho(g) d \nu^{\prime}(g H) \\
= & \frac{\rho(e)}{\chi_{\eta, \xi}} \int_{G / H}\langle\varrho(g H) \xi, \varrho(g H) \eta\rangle \Theta(g H) d \nu^{\prime}(g H) \\
= & \frac{\rho(e)}{\chi_{\eta}, \xi}\langle\xi, \eta\rangle\|\Theta\|_{1} .
\end{aligned}
$$

In the next theorem, we show that the localization operator $\Upsilon_{\Theta, \eta, \xi}$ is compact.

Theorem 3.3 Let $\Theta \in L^{p}(G / H), 1 \leq p<\infty$. Then $\Upsilon_{\Theta, \eta, \xi}: \mathcal{H} \longrightarrow \mathcal{H}$ is a compact operator.

Proof Let $\Theta \in L^{p}(G / H)$ with a G-invariant measure $v^{\prime}$. There exists $\Theta_{n} \in C_{c}(G / H)$ such that $\Theta_{n}-\Theta \longrightarrow 0$. Let $\left\{\eta_{k} ; k=1,2, \ldots\right\}$ be an orthonormal basis for $\mathcal{H}$. Then by Fubini's Theorem and the Schwartz inequality, we have

$$
\begin{aligned}
\Sigma_{k=1}^{\infty} \mid\left\langle\Upsilon_{\Theta_{n}, \eta, \xi} \eta_{k}, \Upsilon_{\Theta_{n}, \eta, \xi} \eta_{k}\right\rangle \\
\leq \frac{1}{\left|\chi_{\eta, \xi}\right|} \Sigma_{k=1}^{\infty} \int_{G / H} \kappa\left(g^{-1}, g H\right)\left|\Theta_{n}(g H)\right|\left\langle\eta_{k}, \varrho(g H) \eta\right\rangle \mid \\
\quad\left|\left\langle\varrho(g H) \xi, \Upsilon_{\Theta_{n}, \eta, \xi} \eta_{k}\right\rangle\right| \rho(g) d \nu^{\prime}(g H) \\
\leq \frac{\rho(e)}{\left|\chi_{\eta, \xi}\right|} \int_{G / H}\left|\Theta_{n}(g H)\right|\left(\Sigma_{k=1}^{\infty}\left|\left\langle\eta_{k}, \varrho(g H) \eta\right\rangle\right|^{2}\right)^{\frac{1}{2}} \\
\quad\left(\Sigma_{k=1}^{\infty}\left|\left\langle\Upsilon_{\Theta_{n}, \eta, \xi}^{*} \varrho(g H) \xi, \eta_{k}\right\rangle\right|^{2}\right)^{\frac{1}{2}} d \nu^{\prime}(g H) \\
\leq \frac{\rho(e)}{\left|\chi_{\eta, \xi}\right|}\left\|\Upsilon_{\Theta_{n}, \eta, \xi}^{*}\right\| \Theta_{n} \|_{1}<\infty .
\end{aligned}
$$

Thus $\Upsilon_{\Theta_{n}, \eta, \xi}$ is in $S_{2}$ and it implies that $\Upsilon_{\Theta_{n}, \eta, \xi}$ is compact. Since

$$
\left\|\Upsilon_{\Theta, \eta, \xi}-\Upsilon_{\Theta_{n}, \eta, \xi}\right\| \leq \frac{\rho(e)^{\frac{1}{p}}}{\left|\chi_{\eta, \xi}\right|}\left(\chi_{\eta} \chi_{\xi}\right)^{\frac{1}{2}\left(1-\frac{1}{p}\right)}\left\|\Theta-\Theta_{n}\right\|_{p}
$$

Then the two-wavelet localization operator is compact.

Acknowledgements The authors would like to thank the referee for their valuable comments and remarks.

Open Access This article is licensed under a Creative Commons Attribution 4.0 International License, which permits use, sharing, adaptation, distribution and reproduction in any medium or format, as long as you give appropriate credit to the original author(s) and the source, provide a link to the Creative Commons licence, and indicate if changes were made. The images or other third party material in this article are included 
in the article's Creative Commons licence, unless indicated otherwise in a credit line to the material. If material is not included in the article's Creative Commons licence and your intended use is not permitted by statutory regulation or exceeds the permitted use, you will need to obtain permission directly from the copyright holder. To view a copy of this licence, visit http://creativecommons.org/licenses/by/4.0/.

\section{References}

1. Benyi, A., Gröchenig, K., Heil, C., Okoudjou, K.: Modulation spaces and a class of bounded multilinear pseudodifferential operators. J. Oper. Theory 54, 389-401 (2005)

2. Catana, V.: Two wavelet localization operators on homogeneous spaces and their traces. Integr. Equ. Oper. Theory 62, 351-363 (2008)

3. Cordero, E., Gröchenig, K.: Necessary conditions for Schatten class localization operators. Proc. Am. Math. Soc. 133, 3573-3579 (2005)

4. Esmaeelzadeh, F., Gol, R.A.K., Tousi, R.R.: On the two-wavelet localization operators on homogeneous spaces with relatively invariant measure. Wavelet Linear Algebra 4(2), 1-12 (2017)

5. Esmaeelzadeh, F., Gol, R.A.K., Tousi, R.R.: On the continuous wavelet transform on homogeneous spaces. Int. J. Wavelets Multiresolut. Inf. Process. (10) 118(4), 1-18 (2012)

6. Folland, G.B.: A Course in Abstract Harmonic Analysis. CRC Press, Boca Raton (1995)

7. Gol, R.A.K., Esmaeelzadeh, F., Tousi, R.R.: Localization operators on homogeneous spaces. Bull. Iranian Math. Soc. 39(3), 455-467 (2013)

8. Gol, R.A.K., Esmaeelzadeh, F., Tousi, R.R.: Two-wavelet constants for square integrable representations of $G / H$. J. Wavelet Linear Algebra 3, 63-73 (2014)

9. Reiter, H., Stegeman, J.: Classical Harmonic Analysis and Locally Compact Group. Claredon Press, Oxford (2000)

10. Rungrottheera, W., Schulze, B.-W., Wong, M.W.: Iterative properties of pseudo-differential operators on edge spaces. J. Pseudo-Differ. Oper. Appl. 5, 455-479 (2014)

11. Rad, O.D.S., Esmaeelzadeh, F., Gol, R.A.K.: On the operator related to CWT on general homogeneous spaces. J. Pseudo-Differ. Oper. Appl. 8, 203-212 (2017)

12. Wong, M.W.: Wavelet Transform and Localization Operators. Birkhäuser Verlag, Basel (2002)

13. Zhu, K.: Operator Theory in Function Spaces. Mathematical Surveys and Monographs, vol. 138. American Mathematical Society, Providence (2007)

Publisher's Note Springer Nature remains neutral with regard to jurisdictional claims in published maps and institutional affiliations. 